INPLASY

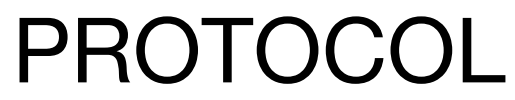

To cite: Torres-Castro et al. Respiratory muscle training in patients with obstructive sleep apnea: A systematic review and meta-analysis. Inplasy protocol 202220096. doi: 10.37766/inplasy2022.2.0096

Received: 22 February 2022

Published: 22 February 2022

Corresponding author:

Rodrigo Torres-Castro

klgorodrigotorres@gmail.com

Author Affiliation:

University of Chile.

Support: None.

Review Stage at time of this submission: The review has not yet started.

Conflicts of interest:

None declared.

\section{Respiratory muscle training in patients with obstructive sleep apnea: A systematic review and meta-analysis}

Torres-Castro, R ${ }^{1}$; Solis-Navarro, L2; Puppo, H33; Vera-Uribe, R4; Alcaraz-Serrano, V5; Vilaró, J6.

Review question / Objective: Is respiratory muscle training effective in patients with obstructive sleep apnea?

Condition being studied: Effects of respiratory muscle training in patients with obstructive sleep apnea.

Information sources: We included the following databases: Pubmed, CENTRAL, Web of Science, CINAHL, EMBASE, Scopus and Scielo. Additionally, the references list of the included studies will be manually reviewed.

INPLASY registration number: This protocol was registered with the International Platform of Registered Systematic Review and Meta-Analysis Protocols (INPLASY) on 22 February 2022 and was last updated on 22 February 2022 (registration number INPLASY202220096).

\section{INTRODUCTION}

Review question / Objective: Is respiratory muscle training effective in patients with obstructive sleep apnea?

Condition being studied: Effects of respiratory muscle training in patients with obstructive sleep apnea.

\section{METHODS}

Participant or population: Adults with obstructive sleep apnea.

Intervention: Respiratory muscle training.

Comparator: No intervention or placebo. 
Study designs to be included: Randomised controlled trials.

Eligibility criteria: 1. Patients with a diagnosis of obstructive sleep apnea diagnosed by a sleep study; 2 . Use of a training device that allows the programming of an objective load.

Information sources: We included the following databases: Pubmed, CENTRAL, Web of Science, CINAHL, EMBASE, Scopus and Scielo. Additionally, the references list of the included studies will be manually reviewed.

Main outcome(s): 1. Apnea/hypopnea index; 2. Epworth sleepiness scale; 3. Pittsburgh sleep quality index; 4. Physical capacity (assessed by standardised tests such as six-minute walk test or cardiopulmonary exercise testing); 5 . Maximal inspiratory pressure (MIP); 6. Maximal expiratory pressure (MEP).

Quality assessment / Risk of bias analysis: The methodological quality assessment framework for RCTs developed by The Cochrane Collaboration's tool for assessing the risk of bias in randomized trials (Higgins and Green 2009) will be used to assess the risk of bias with regards to sequence generation; allocation concealment; blinding (participants, study personnel and outcome assessors); incomplete outcome data; selective outcome reporting; and other sources of potential bias not covered by the framework. To minimize the bias, the studies will be graded independently by 2 reviewers (RTC-LSN). The scoring will be compared and discrepancies will be sorted by a third reviewer (RVU).

Strategy of data synthesis: We will conduct the bibliographic search and compile the identified records in Rayyan QCRI (Rayyan QCRI software, Qatar Computing Research Institute (Data Analysis), Doha, Qatar). All studies identified by the search strategy will be screened against the eligibility criteria by two reviewers (RTC-LSN) working independently based on title and abstract. We will remove any duplicated study. Two authors (RTC-LSN) will work independently to extract data from included studies. A standardised, prepiloted data-extraction form will be used to extract data from included studies. The extracted information will include the author(s), year of study, country, study population characteristics (e.g. population size, age, gender, anthropometric measures), apnea-hypopnea index, maximal inspiratory pressure, maximal expiratory pressure, training device, frequency of training, and duration of the program. Any discrepancies will be discussed or a third reviewer will be asked to adjudicate (HP) Study authors will be contacted with a request for missing data in the included studies.

Subgroup analysis: By type of training (inspiratory vs expiratory).

Sensitivity analysis: We will perform sensitivity analysis based on sample size, heterogeneity, methodological quality, and statistical model. We will exclude studies with low quality, and ensure the stability of analysis results.

\section{Language: English.}

Country(ies) involved: Chile, Spain.

Keywords: Obstructive sleep apnea; respiratory muscle training.

Dissemination plans: We will publish in a peer-reviewed journal.

Contributions of each author:

Author 1 - Rodrigo Torres-Castro.

Author 2 - Lilian Solis-Navarro.

Author 3 - Homero Puppo.

Author 4 - Roberto Vera-Uribe.

Author 5 - Victoria Alcaraz-Serrano.

Author 6 - Jordi Vilaró. 